\title{
Food allergen communication in businesses feasibility trial
}

\author{
Area of research interest: Food hypersensitivity
}

The primary aim of this trial was to investigate whether a larger-scale trial is feasible in the United Kingdom.

The secondary aim was to investigate the likely impact of the intervention, particularly whether proactive allergen communication increased perceptions of food safety and trust of customers, for both those with and without allergies or intolerances.

A multinational food business operator (FBO) was recruited for the trial with 10 branches chosen for participation and split into two groups: the treatment group and the control group.

The treatment group involved staff proactively asking their customers if they had a food allergy at the point of sale. In the control group, customers had to ask for allergen information.

All customers were asked to complete a short survey on a tablet which included questions about perceptions of food safety, trust in the FBO, confidence about asking for allergen information, and overall satisfaction.

Fieldwork was cut short prematurely due to COVID-19. However, the limited findings suggest that the intervention had a positive effect on trust in the businesses and overall satisfaction for those suffering from allergies or intolerances.

The results of the feasibility trial suggest that a fully powered trial would be feasible in the UK if two primary challenges were addressed. Firstly, securing the participation of FBOs, which was challenging despite initial enthusiasm from businesses. Secondly, ensuring the control group do not deliver the intervention, further briefing would be required.

Research report

PDF

View Food allergen communication in businesses feasibility trial as PDF(Open in a new window) $(1.21 \mathrm{MB})$ 